Chronic Obstructive Pulmonary Diseases:

Journal of the COPD Foundation

COPD

rovuntiflon

\author{
Original Research
}

\title{
The Short-term Impact of Symptom-defined COPD Exacerbation Recovery on Health Status and Lung Function
}

\author{
Lindsey T. Murray, $\mathrm{PhD}^{1}$ Nancy K. Leidy, $\mathrm{PhD}^{1}$
}

\begin{abstract}
Background: This study examined the short-term effects of symptom-defined exacerbation recovery on health status and pulmonary function in moderate to severe chronic obstructive pulmonary disease (COPD) patients.

Methods: Secondary analyses of pooled data from two 12-week Phase II international, randomized controlled trials using the EXAcerbations of Chronic Pulmonary Disease Tool (EXACT) to identify symptom-defined exacerbations were conducted. Recovery was categorized as recovered, unrecovered (persistent worsening), or censored. Multiple regression analyses were used to test the effect of recovery status on change in the St George's Respiratory Questionnaire (SGRQ) and forced expiratory volume in 1 second ( $F E V_{1}$ ) from baseline to Week 12. Evaluating Respiratory Symptom scale (E-RS) scores were used to evaluate change in stable-state respiratory symptoms from baseline to Week 12.

Results: Of 1346 eligible patients, 414 (31\%) experienced $\geq 1$ symptom-defined exacerbation; 260 patients recovered from their events, 80 experienced an unrecovered event (persistent worsening), 74 patients had only censored events (excluded). Groups were similar at baseline, with the recovered group reporting significantly worse symptoms $(p<0.01)$. Recovery group and baseline SGRQ were significant predictors of change in health status over 12 weeks ( $p=0.04 ; p<0.01$ ); no effects were observed for lung function. Significant between-group differences in change in respiratory symptom severity from baseline to Week 12 were observed $(p<0.01)$, with the persistent worsening group experiencing clinically meaningful deterioration in breathlessness and chest symptoms.

Conclusions: Results suggest some patients have difficulty recovering from symptom-defined exacerbations, leading to a deterioration in health status, dyspnea, and chest symptoms without short-term effects on lung function. Further study of symptom-defined exacerbation recovery and health outcomes is warranted.
\end{abstract}

Abbreviations: chronic obstructive pulmonary disease, COPD; EXAcerbations of Chronic Pulmonary Disease Tool, EXACT; St George's Respiratory Questionnaire, SGRQ; forced expiratory volume in 1 second, FEV1; Evaluating Respiratory Symptom scale, E-RS; health care resource utilization, HCRU; peak expiratory flow, PEF; respiratory symptom, RS; minimal important difference, MID

Funding Support: Data were provided by AstraZeneca. This research was funded, in part, by the Evidera EXACT-PRO Research \& Development Fund.

Date of Acceptance: October 24, 2017

Citation: Murray LT, Leidy NK. The short-term impact of symptom-defined COPD exacerbation recovery on health status and lung function. Chronic Obstr Pulm Dis. 2018;5(1):27-37. doi: https://doi.org/10.15326/jcopdf.5.1.2017.0166

\section{This article contains an online supplement.}

1 Evidera, Bethesda, Maryland

\section{Keywords:}

COPD; exacerbations; EXACT; patient-reported outcomes;

outcome measures; health status; lung function
Address correspondence to:

Lindsey Murray, PhD

7101 Wisconsin Avenue

Suite 1400

Bethesda, MD

20814

Phone: 301-654-9729

email: lindsey.murray@evidera.com 


\section{Introduction}

Exacerbations of chronic obstructive pulmonary disease (COPD) are events defined by an acute, sustained worsening of the patient's underlying condition of COPD from the stable state beyond normal day-today variability. ${ }^{1,2}$ For the purposes of intervention and natural history studies, exacerbations are classified as health care resource utilization (HCRU) or symptom-defined events. ${ }^{3,4}$ HCRU events include clinic or emergency department visits and treatment with antibiotics and/or oral corticosteroids and/ or hospitalization, 4,5 and account for the majority of the COPD-related burden on health care systems worldwide. ${ }^{1,6-10}$ Symptom-defined exacerbations are assessed through patient-completed diaries. ${ }^{5,11}$ As many as $50 \%-80 \%$ of these events are unreported. ${ }^{12-15}$ Although often described as "mild," symptom severity levels are similar to those observed in reported HCRU exacerbations, and there is evidence to suggest they can adversely affect patient health status and lung function. ${ }^{16-18}$

Research on patterns of recovery from symptomdefined events is limited. There is reason to believe that some patients do not fully recover from one or more of these events, i.e., they fail to return to their previous health. Relatively little is known about the prevalence and nature of these sustained events and their shortand long-term effects. ${ }^{14,17,19-21}$ A natural history study conducted by Donaldson and colleagues found extended duration of symptom-defined exacerbations for up to 99 days occurring in $7.3 \%$ of patients, with recovery defined by peak expiratory flow (PEF) rate. These events negatively impacted health status, forced expiratory volume in 1 second $\left(F E V_{1}\right)$, and time to next HCRU exacerbation. ${ }^{17}$ Leidy and colleagues' analyses of two 3-month, randomized, controlled trials found that $14 \%$ and $15 \%$ of symptom-defined exacerbations, respectively, did not meet recovery requirements based on significant sustained improvement in EXAcerbations of Chronic Pulmonary Disease Tool (EXACT ${ }^{\circledR}$ ) scores. ${ }^{14}$ The purpose of this study was to explore symptomdefined exacerbation recovery in these trials and the short-term effects on health status, lung function, and respiratory symptoms in patients with COPD.

\section{Methods}

\section{Study Design, Sample, Procedures}

Secondary analyses were performed on data from two 12-week, Phase II randomized, double-blind, placebocontrolled multinational trials testing AZD9668 (a neutrophil elastase inhibitor) in patients with COPD. Methods and results are reported elsewhere. ${ }^{22,23}$ Briefly, one trial (referred to here as AZ1) was doseranging with patients standardized to a baseline treatment of tiotropium (Protocol D0520C00012; ClinicalTrials.gov Identifier NCT00949975). The second trial, AZ2, tested one dose against placebo, with patients standardized to a baseline treatment of budesonide/formoterol (Protocol D0520C00020; ClinicalTrials.gov Identifier NCT01023516). Relevant inclusion criteria included: Age 40-80 years; FEV $1 \%$ predicted $40 \%-80 \%$ (AZ1) or $30 \%-80 \%$ (AZ2); $\geq 1$ HCRU visits the prior year; Breathlessness, Cough, and Sputum Scale score $\geq 2$ per day for 7 of 14 days before randomization ${ }^{24,25}$; and no HCRU visits or treatment for exacerbation for at least 4 weeks prior to randomization. No statistically significant differences in lung function or symptom improvement were observed in these trials, ${ }^{22,23}$ permitting pooled analyses across treatment and placebo groups for exploratory purposes. In addition, similar study design and execution allowed for pooling of the 2 datasets for this study.

According to the study sponsor, trial procedures adhered to the Declaration of Helsinki, and the institutional review boards of the participating institutions approved the protocol(s).

\section{Measures}

\section{Patient-reported Measures}

\section{EXACT}

Participants completed an electronic diary each evening, including the EXACT and trial-specific assessments, for the 14-day run-in period leading up to randomization as well as the 12 -week duration of the study. The EXACT consists of 14 questions asking patients to rate severity of breathlessness, cough, sputum, chest congestion, sleep disturbance, feelings of weakness or tiredness, and feelings of worry or concern. ${ }^{26}$ The EXACT score, representing the patient's COPD health state, is tallied each day and ranges from 0-100, with higher scores indicating a more severe condition. ${ }^{27}$

Changes in the daily EXACT score are used to identify symptom-defined exacerbations and quantify symptom severity of HCRU exacerbations. ${ }^{14,26-28}$ Symptom-defined exacerbations are a sustained 
worsening of EXACT daily scores above baseline, i.e., $\geq 9$ points for 3 consecutive days or $\geq 12$ points for 2 consecutive days. In this study, Day -7 to Day -1 constituted baseline. Exacerbation severity is represented by the maximum observed value during an event. Recovery is defined by an improvement of at least 9 points from the maximum value, sustained for 7 days, using a 3-day rolling average-effectively returning the patient to their pre-event level while accounting for normal day-to-day variability. The first day of sustained improvement is designated the day of recovery. Symptom-defined exacerbations that fail to achieve recovery are labeled persistent worsening events. These scoring algorithms have been tested in multiple datasets against clinician and patient global ratings of change and relative to alternative thresholds. ${ }^{14,28}$

For the analyses performed in this study, patients were classified into groups based on their exacerbation recovery pattern. Those in the persistent worsening group experienced an event without recovery at any time prior to 28 days of the end of the study; unrecovered events with an onset within 28 days of the end of the study were defined as censored. After the onset of a persistent worsening event, no subsequent symptomdefined exacerbations were recorded. Patients who experienced recovered events only or recovered events with subsequent censored events were designated as the recovered group. Patients with censored-only events were excluded from the analysis.

\section{St George's Respiratory Questionnaire for COPD}

At baseline and Week 12, patients completed the St George's Respiratory Questionnaire (SGRQ) for COPD, a validated, shortened version of the SGRQ that contains 40 items assessing symptoms (frequency and severity), activity (activities that cause or are limited by breathlessness), and impacts (social functioning, psychological disturbances resulting from airways disease) to capture and summarize health status in patients with COPD. Scores are equivalent to the full 50-item SGRQ and range from 0-100, with higher scores indicating worse COPD health status. ${ }^{29,30}$ A change of 4 points in the total score represents clinically meaningful improvement or deterioration in health status. ${ }^{31}$

\section{Evaluating Respiratory Symptom Scale in COPD}

The Evaluating Respiratory Symptom scale in COPD
(E-RS: COPD $^{\mathrm{TM}}$ ) total and subscale scores, derived using the 11 respiratory symptom items of the EXACT, measure respiratory symptom severity in the stable state. $^{32,33}$ The respiratory symptom (RS)-Total score ranges from 0-40, the RS-Breathlessness score ranges from 0-17, the RS-Cough and Sputum score ranges from 0-11, and the RS-Chest Symptoms score ranges from 0-12. Higher scores represent worse symptom severity. Responder definitions (minimal important difference [MID] change score) are $\geq 2$ for the RSTotal, $\geq 1$ for the RS-Breathlessness, and $\geq 0.7$ for the RS-Cough and Sputum and RS-Chest Symptom scores, respectively. ${ }^{33,34}$ The E-RS was used to assess specific respiratory symptoms affected by recovery status when patients should be in a stable state, i.e., recovered or (in the case of the persistent worsening group) in a new equilibrium.

\section{Clinic Assessments}

Demographic and medical history variables were collected at baseline. Spirometry was performed at baseline and Week 12. The total number of HCRU events reported by patients over the study period was recorded at Week 12.

\section{Analyses}

SAS/STAT software version 9.3 of the SAS System for PC (SAS Institute, Cary, North Carolina) was used for all analyses. Sample descriptive statistics are presented as a frequency and percentage or mean (SD), depending on their distribution. Betweengroup comparisons were conducted using a $\mathrm{X}^{2}$ test or Student's t-test, as appropriate. A $p$-value of $<0.05$ was considered to be statistically significant.

To examine patterns of day-to-day variability during the peri-exacerbation period, mean daily EXACT scores were plotted by recovery group: 1) beginning 14 days prior to event onset and for the remainder of the study period; and 2) beginning at Day -14 of the baseline period through Day 84 (Week 12) (See the online supplement).

Change from baseline to Week 12 in SGRQ total score and $\mathrm{FEV}_{1}$ and were analyzed using multiple regression, with recovery group (recovered versus persistent worsening) and gender as predictors; age, study (AZ1 or AZ2), treatment allocation, and baseline SGRQ or FEV 1 values, respectively, served as covariates. These covariates were similar to those in the Donaldson study and previous studies examining 
unreported symptom-defined exacerbations with the EXACT. ${ }^{17,18,35}$ Due to the limited number of HCRU exacerbations observed over the study period, HCRU count was not included in the models. Covariates' coefficient, standard error, and 95\% confidence interval are reported as well as the model $\mathrm{R}^{2}$ and Adjusted $\mathrm{R}^{2}$ values to assess the model goodness of fit. To facilitate interpretation of regression analyses, change scores in SGRQ and FEV 1 from baseline to Week 12 were compared using Student's t-test (see the online supplement). The proportion of patients in each group whose health status improved, did not change, or deteriorated was also compared across groups using a $\mathrm{x}^{2}$ test, with groups defined by SGRQ thresholds for clinically meaningful change (see the online supplement). ${ }^{31}$

Change in respiratory symptoms from baseline (Days - 7 to -1 prior to baseline visit) to the end of the observation period (Days -7 to -1 prior to Week 12) were compared between groups using the E-RS: COPD to understand the nature of the deterioration in terms of the characteristic symptoms of COPD: breathlessness, cough and sputum, and chest symptoms.

\section{Results}

\section{Sample}

To be included in these analyses, at least 4 baseline days of valid, non-missing EXACT data (Day -7 to Day -1) and $>80 \%$ of daily data from baseline to final visit or early termination date were required. A total of 1346 patients met minimum data criteria ( $93 \%$ of the total randomized trial population), of whom 414 experienced one or more symptom-defined exacerbation. Of these, 74 patients (17.8\%) were excluded due to censoring of their only exacerbation, yielding an analytical sample size of 340 (see online supplement).

Of the 340 patients, 260 (76.7\%) experienced one or more exacerbations with discrete recovery (the recovery group), while 80 (23.5\%) experienced an event defined as unrecovered (the persistent worsening group). In total, this sample experienced a total of 474 symptom-defined exacerbations over the study period. These 340 patients were demographically and clinically similar to those who experienced no symptom-defined exacerbations during the study ( $\mathrm{n}=932)$ (see online supplement).

Baseline sociodemographic and clinical characteristics of the analytical sample $(\mathrm{N}=340)$ are shown in Table 1 . The 2 recovery groups were similar, with the exception of EXACT and E-RS-Total and subscale scores, with the recovered group reporting significantly higher (more severe) values.

Table 2 summarizes the symptom-defined (EXACT) and HCRU exacerbations by recovery group. Patients in the recovered group experienced significantly larger EXACT score changes from baseline to event onset (15.0 \pm 5.31 versus $12.5 \pm 3.51, p<0.01)$ and more severe events $(60.6 \pm 12.38$ versus $57.5 \pm 13.47, p=0.04)$ than the persistent worsening group.

The proportion of patients experiencing an HCRU exacerbation was not statistically different between groups ( $23 \%$ versus $14 \%, p=N S$ ), although a greater proportion of patients in the recovered group reported more than 2 HCRU exacerbations ( $7 \%$ versus $1 \%$, $p=0.05)$.

\section{EXACT Scores Over Time}

Group mean EXACT scores by recovery group for the 14 days leading up to a symptom-defined exacerbation and the remainder of the study are presented in Figure 1. Patients in the persistent worsening group had lower (less severe) EXACT scores on the days leading up to event onset and lower change scores signaling the event than the recovered group (between-group difference $=2.5$ points, $p<0.01$ ). As noted previously, EXACT scores in the recovered group rose 15.0 (5.31) points from baseline and declined rapidly over the next 12-14 days. In contrast, scores in the persistent worsening group worsened by an average of 12.5 (3.51) points. Although a trend toward recovery was observed, EXACT scores remained elevated above baseline and above the recovered group for the remainder of the study. The between-group change in EXACT score from baseline to Week 12 was statistically significant (Recovered group [n=246]: $-2.1 \pm 8.75$; Persistent Worsening group [n=73]: 4.6 $\pm 7.35, p<0.01$ ).

\section{Recovery Status \& Change in Health Status}

Recovery group was a significant predictor of change in health status (Table 3), controlling for baseline SGRQ score, age, sex, study, and treatment group $(\beta=3.52,95 \%$ CI 0.17 to $6.88, p=0.04)$. Group mean change SGRQ scores by recovery status showed that the health status of the recovered patients improved over the course of the study $(-2.1 \pm 13.87)$ while the persistent worsening group declined (1.5 \pm 13.41$)$ $(p=0.04)$ (see online supplement). 


\section{Table 1. Baseline Sociodemographic and Clinical Characteristics}

\begin{tabular}{|c|c|c|c|}
\hline & $\begin{array}{c}\text { Recovered } \\
(\mathrm{N}=260)\end{array}$ & $\begin{array}{l}\text { Persistent } \\
\text { Worsening } \\
(\mathrm{N}=80)\end{array}$ & $p$-value \\
\hline Age, mean (SD) & 61.7 (8.06) & 61.4 (6.89) & 0.79 \\
\hline Gender, $\%$ male & $191(73.5 \%)$ & $59(73.8 \%)$ & 0.96 \\
\hline Race/ethnicity, n (\%) & & & 0.25 \\
\hline White & $227(87.3 \%)$ & $68(85.0 \%)$ & \\
\hline Asian & $31(11.9 \%)$ & $11(13.8 \%)$ & \\
\hline Hispanic or Latino & $0(0.0 \%)$ & $1(1.3 \%)$ & \\
\hline $\mathbf{F E V}_{\mathbf{1}}(\mathrm{L})$, mean $(\mathrm{SD})$ & $1.6(0.52)$ & $1.6(0.52)$ & 0.56 \\
\hline FEV $_{1} \%$ predicted, mean (SD) & $55.4(14.56)$ & $56.7(15.49)$ & 0.48 \\
\hline GOLD stage (spirometry only), n (\%) & & & 0.92 \\
\hline I & $10(3.9 \%)$ & $4(5.0 \%)$ & \\
\hline II & $137(52.7 \%)$ & $46(57.5 \%)$ & \\
\hline III & $101(38.9 \%)$ & $27(33.8 \%)$ & \\
\hline IV & $4(1.5 \%)$ & $1(1.3 \%)$ & \\
\hline $\begin{array}{l}\text { Number of HCRU Exacerbations in Past } \\
12 \text { months, mean (SD) }\end{array}$ & $1.4(0.79)$ & $1.4(0.75)$ & 0.97 \\
\hline \multicolumn{4}{|l|}{ SGRQ Baseline Scores, mean (SD) ${ }^{a}$} \\
\hline Total & $55.4(17.46)$ & $55.8(16.25)$ & 0.87 \\
\hline Symptoms & $74.6(17.64)$ & $70.7(16.77)$ & 0.08 \\
\hline Activity & $44.5(19.82)$ & $44.6(21.28)$ & 0.95 \\
\hline Impacts & $62.53(21.51)$ & $66.1(17.68)$ & 0.14 \\
\hline EXACT Baseline Score, mean (SD) ${ }^{a, b}$ & $43.5(9.97)$ & $39.6(10.93)$ & $<0.01$ \\
\hline \multicolumn{4}{|l|}{ E-RS Baseline Scores, mean (SD) ${ }^{b, c}$} \\
\hline RS - Total & $16.8(6.41)$ & $14.3(6.84)$ & $<0.01$ \\
\hline RS - Breathlessness & $8.3(3.43)$ & $7.1(3.61)$ & $<0.01$ \\
\hline RS - Cough \& Sputum & $4.4(1.68)$ & $3.7(1.56)$ & $<0.01$ \\
\hline RS - Chest Symptoms & $4.1(1.92)$ & $3.5(2.09)$ & 0.02 \\
\hline AZ Study 1, n (\%) & $148(56.9 \%)$ & $45(56.3 \%)$ & 0.92 \\
\hline \multicolumn{4}{|c|}{ 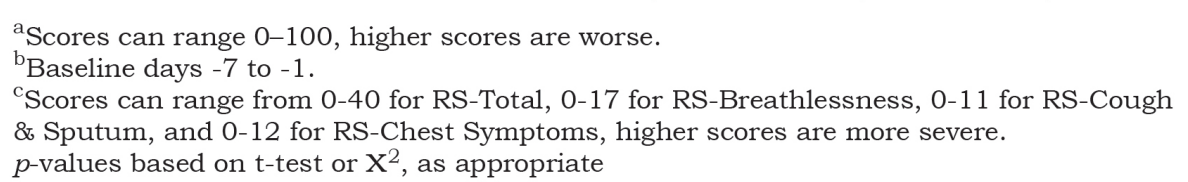 } \\
\hline \multicolumn{4}{|c|}{$\begin{array}{l}\text { AZ=AstraZeneca; EXACT=EXAcerbations of Chronic Pulmonary Disease Tool; } \\
\text { E-RS=Evaluating Respiratory Symptoms scale; RS=respiratory symptoms; FEV } V_{1}=\text { forced } \\
\text { expiratory volume in } 1 \text { second; GOLD=Global Initiative for Obstructive Lung Disease; } \\
\text { HCRU=health care resource utilization; SGRQ=St George's Respiratory Questionnaire. }\end{array}$} \\
\hline
\end{tabular}

\section{Recovery Status \& Change in Lung Function}

There was no relationship between recovery group and change in lung function (Table 3). Baseline FEV 1 was a significant predictor ( $\beta=-0.09,95 \%$ CI -0.15 to -0.04, $p<0.01$ ) (Table 3). Mean FEV 1 change scores at Week 12 were effectively 0 for both the recovered $(-0.03 \pm 0.21)$ and persistent worsening groups
$(-0.04 \pm 0.29) \quad(p=0.89) \quad$ (see online supplement).

\section{Change in Respiratory Symptoms}

Post-hoc analyses to understand the nature of persistent worsening in terms of respiratory symptoms showed statistically significant differences between the 2 recovery groups in change from baseline to end of study (Week 12) for respiratory symptoms overall (E-RS total score) and each of the 3 subscales (breathlessness, cough \& sputum, and chest symptoms) $\quad(p<0.01$ for all). Although the recovered group showed symptomatic improvement, values were less than the proposed MID, suggesting these patients were essentially unchanged from baseline. In contrast, the persistent worsening group experienced meaningful deterioration in respiratory symptoms overall (E-RS-Total), breathlessness (RS-Breathlessness) and chest symptoms (RS-Chest Symptom) (Figure 2).

\section{Discussion}

This study explored the patterns and effects of patient recovery from symptom-defined exacerbations over 12 weeks. Results support previous findings that a subset of patients experience acute worsening of their COPD, characteristic of a symptomdefined exacerbation, that persists over time, rather than returning to preevent or near pre-event levels. These events were associated with a decline in health status and meaningful deterioration in respiratory symptoms over 12 weeks, with no apparent effect on $\mathrm{FEV}_{1}$.

In the current study, $16.9 \%$ of the symptom-defined exacerbations observed were classified as persistent worsening at 12 weeks. This finding is higher than previous studies of symptom-defined exacerbation where recovery was defined as symptom improvement 


\section{Table 2. Health Care Resource Utilization and Symptom-defined Exacerbations During the 12-Week Study by Recovery Group}

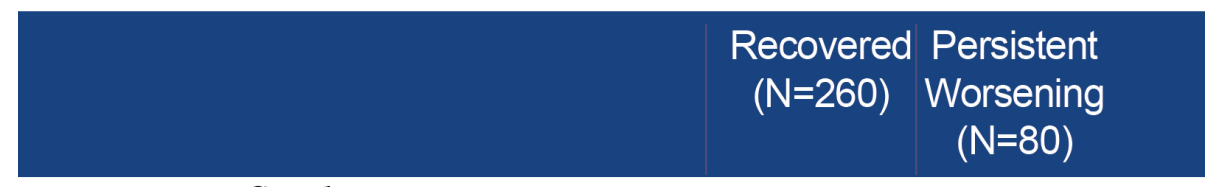

Symptom-Defined Events

\begin{tabular}{|c|c|c|}
\hline Event Frequency, $\mathrm{n}$ [range] ${ }^{\mathrm{a}}$ & $383[1-4]$ & $91[1-2]$ \\
\hline$\geq 2$ Events, $\mathrm{n}(\%)$ & $95(36.8 \%)$ & $11(13.8 \%)$ \\
\hline Median Time to First Event, days ${ }^{b}$ & 26.5 & 27.5 \\
\hline Event Duration, days mean $(\mathrm{SD})^{\mathrm{C}}$ & $12.9(12.3)$ & $\mathrm{n} / \mathrm{a}$ \\
\hline $\begin{array}{l}\text { EXACT Change Score at Onset Day } 1 \text {, } \\
\text { mean }(\mathrm{SD})^{\mathrm{d}}\end{array}$ & $15.0(5.31)$ & $12.5(3.51)$ \\
\hline Event Severity, mean $(\mathrm{SD})^{\mathrm{d}, \mathrm{e}}$ & $60.6(12.38)$ & $57.5(13.47)$ \\
\hline HCRU Events, n (\%) & $59(22.7 \%)$ & $11(13.8 \%)$ \\
\hline Event Frequency, $\mathrm{n}$ [range] $]^{\mathrm{a}}$ & $83[1-4]$ & $12[1-2]$ \\
\hline$\geq 2$ Events, $\mathrm{n}(\%)$ & $18(6.9 \%)$ & $1(1.3 \%)$ \\
\hline Median Time to First HCRU, days & 29.0 & 50.0 \\
\hline Event Duration, days mean (SD) & $9.7(5.53)$ & $7.8(4.09)$ \\
\hline Event Severity, mean $(\mathrm{SD})^{\mathrm{e}, \mathrm{f}}$ & $58.7(11.42)$ & $61.8(11.32)$ \\
\hline
\end{tabular}

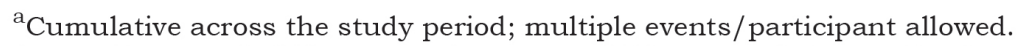
Among Recovered, 329 recovered events and 54 censored. Among Persistent Worsening, 11 recovered events and 80 persistent worsening.

${ }^{\mathrm{b}}$ Time to first resolved event for Recovered group or first persistent worsening event for Persistent Worsening group.

${ }^{\mathrm{c}}$ Excludes 54 events that were censored.

${ }^{d}$ Persistent worsening events only. Excludes 11 first symptom-defined events that recovered.

${ }^{\mathrm{e}}$ Severity defined by the worst EXACT score observed during the event or during the HCRU.

${ }^{\mathrm{f}} \mathrm{N}=77$ events for Recovered group and $\mathrm{N}=10$ events for Persistent Worsening group due to missing EXACT data during HCRU event.

EXACT $=$ EXAcerbations of Chronic Pulmonary Disease Tool; HCRU=health care resource utilization

for 2 consecutive days and rates ranged from $3.1 \%$ to $9.6 \%$ at up to 99 days. ${ }^{17,19,21}$ Differences in nonrecovery rates are likely due, in part, to differences in recovery definitions, with the EXACT requiring sustained symptomatic improvement for 7 consecutive days and a 3-day rolling average accounting for dayto-day variability. This conservative approach assures users that those who recover from these events have returned to a stable state similar to their pre-event status. Results of the current study support the identification and definition of persistent worsening events. They also suggest that, in the absence of treatment, patients experiencing these events are at a new steady state with poorer health status and should have their EXACT scores reset in order to appropriately identify subsequent acute events.

It was interesting to find that patients in the persistent worsening group had significantly lower EXACT scores at baseline and more gradual event onset compared with patients who recovered. This finding is in-line with those reported by Aaron and colleagues, in which patients who experienced event onset on the same day symptoms began had shorter median recovery times to baseline health status compared with patients who experienced gradual onset. $^{36}$ MacKay and colleagues also showed that among symptom-defined exacerbations captured with the EXACT, patients with more severe stable disease (defined by a history of exacerbations in the last year and airflow obstruction) were more likely to report events and receive treatment for symptom-defined exacerbations associated with smaller increases in symptom severity at event onset compared to patients with milder stable disease. ${ }^{37}$ Jones and colleagues also found that patients with unreported-and therefore untreatedsymptom-defined exacerbations had lower EXACT scores at baseline than patients with reported symptom-defined exacerbations, and that EXACT change scores from baseline remained elevated 28 days after the onset of unreported symptom-defined exacerbations compared to treated exacerbations. ${ }^{18}$ Together, these findings suggest patients with lower EXACT scores (e.g., better health status) at baseline, and patients with more gradual symptom deterioration may be less likely to report acute symptomatic events. Symptom-defined exacerbations with a more gradual onset may be more difficult for patients to identify as an acute worsening in their COPD health that is worth a health care visit for assessment and possible treatment. Patients experiencing milder daily symptoms and a gradual rise in symptoms may 
benefit from additional training on how to recognize deteriorating symptoms and encouragement to notify their clinician for treatment.

Findings from this study are consistent with previous studies suggesting symptom-defined exacerbations may have an adverse effect on health

Figure 1. Mean EXACT Scores on Days -14 Relative to Onset of Symptom-defined Exacerbation to End of Study by Recovery Group

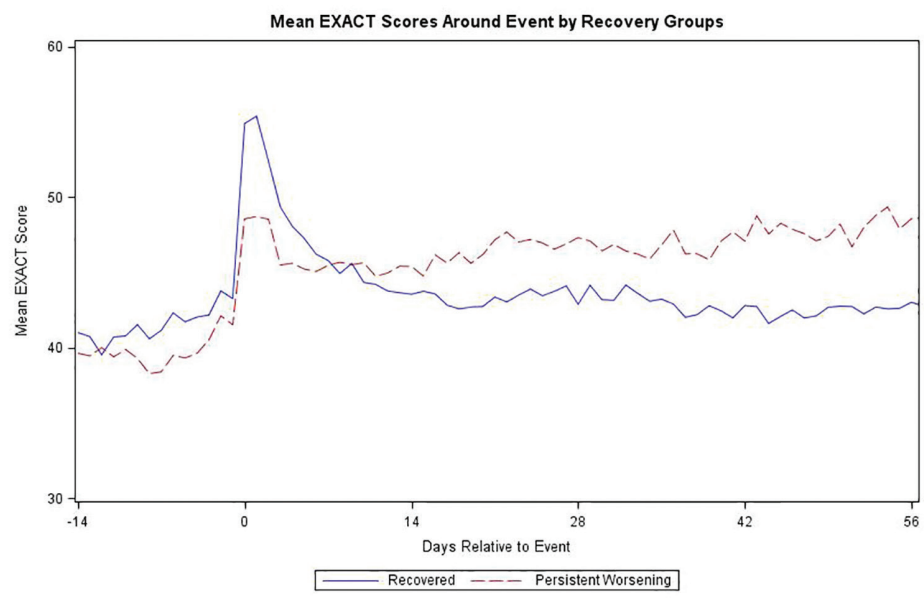

Higher scores are worse.

Recovered Group n=260; Persistent Worsening Group n=80; Patients could contribute multiple events to the mean

EXACT=EXAcerbations of Chronic Pulmonary Disease Tool outcomes. ${ }^{17,18}$ Absolute differences in SGRQ change scores between the recovered and persistent worsening groups approached the published 4-point minimally important difference. ${ }^{31}$ The deterioration in respiratory symptoms from baseline to Week 12 in this group was clinically meaningful. Although it is reassuring to see no effect on lung function in this study, this should not be extrapolated to longer-term effects. Donaldson and colleagues found that patients with one or more non-recovered events (defined as PEF not returning to baseline after 99 days) had an $\mathrm{FEV}_{1}$ decline that was $-10.8 \mathrm{ml} /$ year faster than patients with no non-recovered events over a median of 3 years. ${ }^{17}$ Further study of the relationship between symptom-defined exacerbations, recovery status, and lung function is warranted. Pending results of this work, it is possible that early intervention programs could alleviate patient morbidity in the short term, and allay disease progression in the long term.

Several limitations of this study are worth noting. First, the 3-month study duration limited the evaluation to short-term effects. Second, the small number of HCRU exacerbations precluded the inclusion of this variable in the regression models and may have contributed to the small $\mathrm{R}^{2}$ values. The trend toward more HCRU events in the recovered group (i.e., treatment with antibiotics or steroids during the 12week period) could have contributed to more stable or

\section{Table 3. Effect of Recovery Status on Change in Health Status and Lung Function at 12 Weeks}

\begin{tabular}{|c|c|c|c|c|c|c|c|c|}
\hline \multirow{2}{*}{ Variable } & \multicolumn{4}{|c|}{$\begin{array}{c}\text { Health Status (SGRQ) } \\
\qquad \begin{array}{c}R^{2}=0.12 \\
\text { Adj. } R^{2}=0.11\end{array}\end{array}$} & \multicolumn{4}{|c|}{$\begin{array}{l}\text { Lung Function }\left(F E V_{1}\right) \\
R^{2}=0.04 \\
\text { Adj. } R^{2}=0.02\end{array}$} \\
\hline & $\beta$ & SE B & $\begin{array}{c}95 \% \\
\text { Confidence } \\
\text { Interval }\end{array}$ & $p$-value & $\beta$ & SE B & $\begin{array}{c}95 \% \\
\text { Confidence } \\
\text { Interval }\end{array}$ & $p$-value \\
\hline Symptom-defined Recovery Status & 3.52 & 1.70 & 0.17 to 6.88 & 0.04 & -0.00 & 0.03 & -0.06 to 0.06 & 0.94 \\
\hline Sex & -0.21 & 1.67 & -3.49 to 3.07 & 0.90 & -0.00 & 0.03 & -0.07 to 0.06 & 0.86 \\
\hline Age & -0.07 & 0.09 & -0.26 to 0.11 & 0.43 & 0.00 & 0.00 & -0.00 to 0.00 & 0.90 \\
\hline Study & -2.03 & 1.47 & -4.92 to 0.86 & 0.17 & -0.03 & 0.03 & -0.08 to 0.02 & 0.29 \\
\hline Treatment Group & 0.28 & 0.55 & -0.80 to 1.37 & 0.61 & -0.00 & 0.01 & -0.02 to 0.02 & 0.70 \\
\hline SGRQ Total Baseline Score & -0.26 & 0.04 & -0.35 to -0.18 & $<0.01$ & -- & -- & -- & -- \\
\hline FEV $_{1}$ (L) Baseline & -- & -- & -- & -- & -0.09 & 0.03 & -0.15 to -0.04 & $<0.01$ \\
\hline
\end{tabular}

SGRQ=St George's Respiratory Questionnaire; $\mathrm{FEV}_{1}=$ forced expiratory volume in 1 second 


\section{Figure 2. Summary of Change Scores in Evaluating Respiratory Symptoms Total and Subscale Scores From Baseline to Week $12^{\mathrm{a}}$ by Recovery Group}

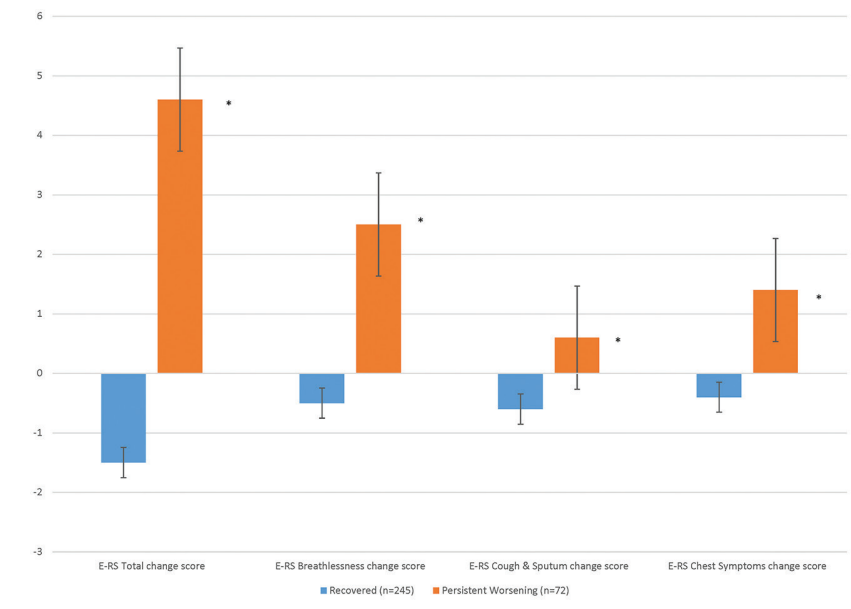

Higher scores are worse.

Responder definitions are $\geq 2$ for the RS-Total, $\geq 1$ for the RS-Breathlessness, and $\geq 0.7$ for the RS-Cough and Sputum and RS-Chest Symptom scores, respectively.

${ }^{a}$ Mean of final week Days -7 to -1 from Week $12 ; 15$ patients in the Recovered group and 8 patients in the Persistent Worsening group had insufficient E-RS data for Week 12 to compute a weekly mean.

${ }^{*} p<0.0001$ for recovered vs. persistent worsening change scores; $p$-values based on t-test

E-RS=Evaluating Respiratory Symptoms tool

improved health status and symptom severity in these patients. Third, the data did not permit an analysis of treatment-seeking proclivities or behaviors. It is possible that the persistent worsening group was less likely to seek care for a change in condition. These results offer support for studies to better understand patient treatment-seeking preferences and the effects of targeted, early intervention.

Several strengths are also noteworthy. First, this was a large dataset from 2 multi-national, randomized, controlled clinical trials. Second, EXACT diary compliance was high. Together, this ensures excellent internal validity since data were collected completely and systematically. Finally, patients were largely representative of symptomatic trial patients in terms of gender, age, and maintenance treatment, with moderate to severe COPD and a history of at least one HCRU in the 12 months prior-making the results of this study applicable to similar COPD patient populations.

\section{Conclusion}

This study explored recovery patterns associated with symptom-defined exacerbations, and assessed the extent to which sustained events affect health status, lung function, and respiratory symptoms over a short 12-week period. Results suggest some patients have difficulty recovering from symptomdefined exacerbations, leading to a decline in health status and increased levels of breathlessness and chest symptoms that may represent an early signal of disease progression. Further study of patient treatmentseeking behaviors and the effects of baseline health status, recognition of symptom deterioration, and early treatment on symptom-defined exacerbation recovery and health outcomes is warranted.

\section{Acknowledgements}

The authors thank AstraZeneca for providing the two 3-month trial datasets (AZ 1 and AZ 2) used in these analyses. They also thank Drs. Heather Young, Naji Younes, and Kimberly Robien from George Washington University Milken Institute for Public Health for their comments on an earlier version of this paper in support of L. Murray's PhD dissertation. The authors would also like to thank Ren Yu, MA, for programming support and Fritz Hamme for production support.

Author Contributions: Each author contributed substantially to the research described in this paper, including contributions to the concept and design, analyses, and interpretation of the data. LT Murray and NK Leidy supervised acquisition of the databases, design and execution of the statistical analysis plans and drafting the manuscript. LT Murray revised the paper in response to co-author and referee comments. All authors reviewed and approved this version of the paper prior to submission. Data Statement: This study included data that have been previously presented. Efficacy analyses for each trial were presented separately by Kuna and colleagues ${ }^{22}$ and Vogelmeier and colleagues. ${ }^{23}$ These data were also used to evaluate the psychometric performance of the EXACT and E-RS tools and reported by Leidy and colleagues. ${ }^{14,33}$

\section{Declaration of Interest}

LT Murray and NK Leidy are employed by Evidera, which provides consulting and other research services to pharmaceutical, device, government and non- 
government organizations. As Evidera employees, they work with a variety of companies and organizations and are expressly prohibited from receiving any payment or honoraria directly from these organizations for services rendered. 


\section{References}

1. Global Initiative for Chronic Obstructive Lung Disease (GOLD). Global strategy for the diagnosis, management and prevention of COPD, 2015. GOLD website. http://www.goldcopd.org. Published 2015. Accessed December 2017.

2. Rodriguez-Roisin R. Toward a consensus definition for COPD exacerbations. Chest. 2000;117(Suppl. 2):398S-401S. doi: https://doi.org/10.1378/chest.117.5_suppl_2.398S

3. Calverley P, Loefdahl C, Svensson K, Higenbottom T, Carlsson L-G, Stahl E. Relationship between respiratory symptoms and medical treatment in exacerbations of COPD. Eur Resp J. 2005;26(3):406-413.

doi: https://doi.org/10.1183/09031936.05.00143404

4. Rennard S, Leidy NK. Definition and severity of COPD exacerbations. In: Wedzicha W, Martinez F, eds. Exacerbations of Chronic Obstructive Pulmonary Disease (COPD). New York: Informa Healthcare; 2009.

5. Celli BR, MacNee W, Agusti A, et al. Standards for the diagnosis and treatment of patients with COPD: A summary of the ATS/ ERS position paper. Eur Resp J. 2004;23(6):932-946.

6. Guarascio A, Ray S, Finch C, Self T. The clinical and economic burden of chronic obstructive pulmonary disease in the USA. Clinicoecon Outcomes Res. 2013; 5:235-245.

7. Mannino DM. COPD: Epidemiology, Prevalence, Morbidity and Mortality, and Disease Heterogeneity. Chest. 2002;121(5, Supplement):121S-126S.

8. Mannino DM, Buist AS. Global burden of COPD: risk factors, prevalence, and future trends. Lancet. 2007; 370(9589):765-773. doi: https://doi.org/10.1016/S0140-6736(07)61380-4.

9. Mathers D, Loncar D. Projections of global mortality and burden of disease from 2002 to 2030. PLoS Med. 2006;3:e442. doi: https:// doi.org/10.1371/journal.pmed.0030442

10. Murray CJL, Lopez AD. Measuring the global burden of disease. New Eng J Med. 2013;369(5):448-457.

doi: https://doi.org/10.1056/NEJMra 1201534

11. Burge S, Wedzicha JA. COPD exacerbations: definitions and classifications. Eur Respir J. 2003;41(Suppl):46s-53s. doi: https://doi.org/10.1183/09031936.03.00078002

12. Langsetmo L PR, Ernst P, Bourbeau J. Underreporting exacerbation of chronic obstructive pulmonary disease in a longitudinal cohort. Am J Respir Crit Care Med. 2008;177(4):396401. doi: https://doi.org/10.1164/rccm.200708-12900C

13. Xu W, Collet J-P, Shapiro S, et al. Negative impacts of unreported COPD exacerbations on health-related quality of life at 1 year. Eur Respir J. 2010;35(5):1022-1030.
14. Leidy NK, Murray LT, Jones P, Sethi S. Performance of the EXAcerbations of Chronic pulmonary disease Tool patient-reported outcome measure in three clinical trials of chronic obstructive pulmonary disease. Ann Am Thorac Soc. 2014;11(3):316-325.

doi: https://doi.org/10.1513/AnnalsATS.201309-305OC

15. Seemungal T, Donaldson G, Paul E, Bestall J, Jeffries D, Wedzicha J. Effect of exacerbation on quality of life in patients with chronic obstructive pulmonary disease. Am J Respir Crit Care Med. 1998;157(5 PT 1):1418-1422.

doi: https://doi.org/10.1164/ajrccm.157.5.9709032

16. Sato M, Chubachi S, Sasaki M, et al. Impact of mild exacerbation on COPD symptoms in a Japanese cohort. Int J Chron Obstruct Pulmon Dis. 2016;11:1269-1278.

17. Donaldson G, Law M, Kowlessar B, et al. Impact of prolonged exacerbation recovery in chronic obstructive pulmonary disease. Am J Respir Crit Care Med. 2015;192(8):943-950. doi: https://doi.org/10.1164/rccm.201412-22690C

18. Jones PW, Lamarca R, Chuecos F, et al. Characterisation and impact of reported and unreported exacerbations: results from ATTAIN. Eur Respir J. 2014;44(5):1156-1165. doi: https://doi.org/10.1183/09031936.00038814

19. Donaldson G, Seemungal T, Patel I, Lloyd-Owen S, Wilkinson $\mathrm{T}$, Wedzicha J. Longitudinal changes in the nature, severity and frequency of COPD exacerbations. Eur Respir J. 2003 2003;22(6):931-936.

20. Miravitlles M, Garcia-Sidro P, Fernandez-Nistal A, Buendia M, de los Monteros M, Molina J. Course of COPD assessment test (CAT) and clinical COPD questionnaire (CCQ) scores during recovery from exacerbations of chronic obstructive pulmonary disease. Health Qual Life Outcomes. 2013;11:147. doi: https://doi.org/10.1186/1477-7525-11-147

21. Seemungal T, Donaldson G, Bhowmik A, Jeffries D, Wedzicha $\mathrm{J}$. Time course and recovery of exacerbations in patients with chronic obstructive pulmonary disease. Am J Respir Crit Care Med. 2000;161:1608-1613.

doi: https://doi.org/10.1164/ajrccm.161.5.9908022

22. Kuna P, Jenkins M, O’Brien C, Fahy W. AZD9668, a neutrophil elastase inhibitor, plus ongoing budesonide/formoterol in patients with COPD. Respir Med. 2012;106:531-539. doi: https://doi.org/10.1016/j.rmed.2011.10.020

23. Vogelmeier C, Aquino T, O’Brien C, Perrett J, Gunawardena K. A randomized, placebo-controlled, dose-finding study of AZD9668, an oral inhibitor of neutrophil elastase, in patients with chronic obstructive pulmonary disease treated with tiotropium. COPD. 2012;9:111-120. doi: https://doi.org/10.3109/15412555.2011.641803 
24. Leidy N, Rennard S, Schmier J, Jones M, Goldman M. The breathlessness, cough, and sputum scale: the development of empirically based guidelines for interpretation. Chest. 2003; 124(6):2181-2191.

doi: https://doi.org/10.1378/chest.124.6.2182

25. Leidy N, Schmier J, Jones M, Lloyd J, Rocchiccioli K. Evaluating symptoms in chronic obstructive pulmonary disease: validation of the Breathlessness, Cough and Sputum Scale. Respir Med. 2003;97(Supple A):S59-S70.

doi: https://doi.org/10.1016/S0954-6111(03)80016-1

26. Leidy $\mathrm{N}$, Wilcox $\mathrm{T}$, Jones $\mathrm{P}$, et al. Development of the EXAcerbations of Chronic Obstructive Pulmonary Disease Tool (EXACT): A Patient-reported outcome (PRO) measure. Value Health. 2010;13(8):965-975.

doi: https://doi.org/10.1111/j.1524-4733.2010.00772.x

27. Jones PW, Chen WH, Wilcox TK, Sethi S, Leidy NK. Characterizing and quantifying the symptomatic features of COPD exacerbations. Chest. 2011;139(6):1388-1394.

doi: https://doi.org/10.1378/chest.10-1240

28. Leidy N, Wilcox T, Jones P, et al. Standardizing measurement of chronic obstructive pulmonary disease exacerbations: reliability and validity of a patient-reported diary. Am J Respir Crit Care Med. 2011;183(3):323-329.

doi: https://doi.org/10.1164/rccm.201005-0762OC

29. Jones PW, Quirk FH, Baveystock CM, Littlejohns P. A selfcomplete measure of health status for chronic airflow limitation. The St George's Respiratory Questionnaire. Am Rev Respir Dis. 1992;145(6): 1321-1327.

doi: https://doi.org/10.1164/ajrccm/145.6.1321

30. Meguro M, Barley E, Spencer S, Jones P. Development and validation of an improved COPD-specific version of the St. George's Respiratory Questionnaire. Chest. 2006;132:456-463. doi: https://doi.org/10.1378/chest.06-0702

31. Jones P. Interpreting thresholds for a clinically significant change in health status in asthma and COPD. Eur Respir J. 2002;19(3):398-404.

32. Leidy NK, Sexton CC, Jones PW, et al. Measuring respiratory symptoms in clinical trials of COPD: reliability and validity of a daily diary. Thorax. 2014;69(5):443-449.

doi: https://doi.org/10.1136/thoraxjnl-2013-204428

33. Leidy NK, Murray LT, Monz BU, et al. Measuring respiratory symptoms of COPD: performance of the EXACT- Respiratory Symptoms Tool (E-RS) in three clinical trials. Respir Res. 2014;15:124. doi: https://doi.org/10.1186/s12931-014-0124-z

34. Jones PW, Leidy NK, Hareendran A, Lamarca R, Chuecos F, Garcia Gil E. The effect of aclidinium bromide on daily respiratory symptoms of COPD, measured using the Evaluating Respiratory Symptoms in COPD (E-RS: COPD) diary: pooled analysis of two 6-month Phase III studies. Respir Res. 2016;17(1):61.

doi: https://doi.org/10.1186/s12931-016-0372-1
35. Ehsan M, Khan R, Wakefield D, et al. A longitudinal study evaluating the effect of exacerbations on physical activity in patients with chronic obstructive pulmonary disease. Ann Am Thorac Soc. 2013;10(6):559-564.

doi: https://doi.org/10.1513/AnnalsATS.201304-100OC

36. Aaron SD, Donaldson GC, Whitmore GA, Hurst JR, Ramsay T, Wedzicha JA. Time course and pattern of COPD exacerbation onset. Thorax. 2012;67(3):238-243.

37. Mackay AJ, Donaldson GC, Patel ARC, Singh R, Kowlessar $\mathrm{B}$, Wedzicha JA. Detection and severity grading of COPD exacerbations using the exacerbations of chronic pulmonary disease tool (EXACT). Eur Respir J. 2014;43(3):735-744. 